\title{
Neoplasia ovárica (Luteoma) en una Suricata (Suricata suricatta)
}

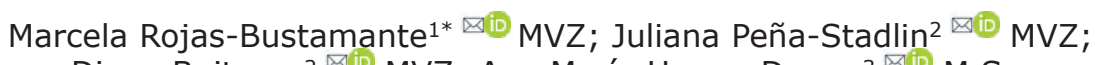
Diana Buitrago $2 \bowtie(\mathbb{0})$ MVZ; Ana María Henao-Duque ${ }^{3 凶(0)}$ M.Sc.

\author{
1Universidad CES, Facultad de medicina veterinaria y zootecnia, Medellín, Colombia. \\ ${ }^{2}$ Fundación Zoológica de Cali, Unidad de Bienestar animal, Cali, Colombia. \\ ${ }^{3}$ Zoopath, Laboratorio de Patología veterinaria, Cali, Colombia. \\ *Correspondencia: marcelarojas0998@gmail.com
}

Recibido: Mayo 2020; Aceptado: Diciembre 2020; Publicado: Abril 2021.

\section{RESUMEN}

En este artículo se describe el caso de una Suricata suricatta de 5 años, hembra, nacida bajo cuidado humano en el Zoológico de Cali, Colombia, que presentó reporte de comportamiento anormal. Al examen clínico se observó alopecia multifocal y simétrica bilateral con costras. Se realizó una evaluación ultrasonográfica que determinó la presencia de quistes ováricos. Como alternativa terapéutica se realizó una ovariohisterctomía (OVH) con biopsia escisional de ambos ovarios, que fueron remitidos al laboratorio de patología. Los hallazgos histopatológicos concluyeron una neoplasia del ovario izquierdo consistente con un Luteoma. El ovario derecho no presentó lesiones significativas. Durante la cirugía se tomaron muestras de sangre que indicaron que el paciente presentaba anemia regenerativa, química sanguínea con aumento del BUN y proteínas totales asociados a una hiperglobulinemia indicativos de un proceso inflamatorio crónico. La paciente se recuperó satisfactoriamente de la cirugía y tras dos semanas posteriores al procedimiento, las lesiones dermatológicas remitieron, así como los problemas de comportamiento previamente reportados.

Palabras clave: Herpestidae; OVH; problemas hormonales; quistes ováricos; zoológico; ultrasonografía (Fuentes: DeCS, MeSH).

\section{ABSTRACT}

This article describes the case of a 5-year-old female meerkat born under human care at the Cali Zoo in Colombia, which presented a report of abnormal behavior. Upon clinical examination, multifocal and symmetrical bilateral scabbed alopecia was observed. An ultrasonographic evaluation was carried out determining the presence of ovarian cysts. As a therapeutic alternative, an ovariohysterectomy $(\mathrm{OVH})$ was performed with an excisional biopsy of both ovaries, which were sent to the pathology laboratory. The histopathological findings concluded a neoplasm of the left ovary consistent with a Luteoma. Nonetheless, the right ovary did not present significant lesions. During the surgery, blood samples were taken which indicated that the patient presented regenerative anemia, blood

Como citar (Vancouver).

Rojas-Bustamante M, Peña-Stadlin J, Buitrago D, Henao-Duque AM. Neoplasia ovárica (Luteoma) en una Suricata (Suricata suricatta). Rev MVZ Córdoba. 2021; 26(2):e1994. https://doi.org/10.21897/rmvz.1994 
chemistry with increased BUN and total proteins associated with hyperglobulinemia indicative of a chronic inflammatory process. The patient recovered satisfactorily from the surgery and after two weeks following the procedure, the dermatological lesions receded, as well as the behavioral problems previously reported.

Keywords: Herpestidae; OVH; hormonal problems; ovarian cysts; zoo; ultrasonography (Sources: $\mathrm{DeCS}, \mathrm{MeSH}$ ).

\section{INTRODUCCIÓN}

La especie Suricata es una de las 35 especies de mangostas de la familia Herpestidae y del género Suricata. Las suricatas son denominadas comúnmente como Suricate, Suricata Gris, Suricata de cola delgada. Según la IUCN (International union for conservation of nature) son animales catalogados como de menor preocupación en la lista roja y su población se encuentra estable (1). Son pequeños carnívoros que viven en condiciones desérticas y son estrictamente diurnos. Tienen una expectativa de vida en vida libre de 10 años y bajo cuidado humano, de 14 años. (2)

Las afecciones del sistema reproductivo son comunes en múltiples especies animales, sin embargo, las neoplasias ováricas no se diagnostican con frecuencia a causa de su naturaleza subclínica (3); y los reportes de estas en suricatas son escasos. Las afecciones comúnmente presentadas en estos animales que viven bajo cuidado humano son enfermedades infecciosas principalmente toxoplasmosis $(4,5)$, patologías degenerativas, casos de colesteatoma en suricatas, uno de ellos asociado a arterosclerosis (6) y en raras ocasiones tumores (4). Por esta razón, se describe este reporte de caso, ya que se espera que el monitoreo de este tipo de afecciones se tenga en cuenta en los abordajes clínicos de medicina preventiva realizados en esta especie.

\section{DESCRIPCION DEL CASO}

Anamnesis. Una hembra de 5 años nacida bajo cuidado humano en el Zoológico de Cali, que compartía el recinto con un macho, presentó reporte de múltiples apareamientos dos meses atrás, razón por la que fue sometida a un examen clínico y evaluación ecográfica con el fin de determinar si se encontraba en estado de gestación.

Hallazgos a la evaluación física. Se realizó un examen clínico mediante restricción física, en el cual se observó pelaje hirsuto y seborreico, además de alopecia e hipotricosis multifocal en la comisura labial (Figura $1 \mathrm{~A}$ ) y en el dorso hacia caudal con distribución simétrica bilateral y costras (Figura 1B), adicional a estas lesiones se reportó que el animal presentaba signos de dolor, presentando una marcada cifosis al desplazarse, además de una baja condición corporal.

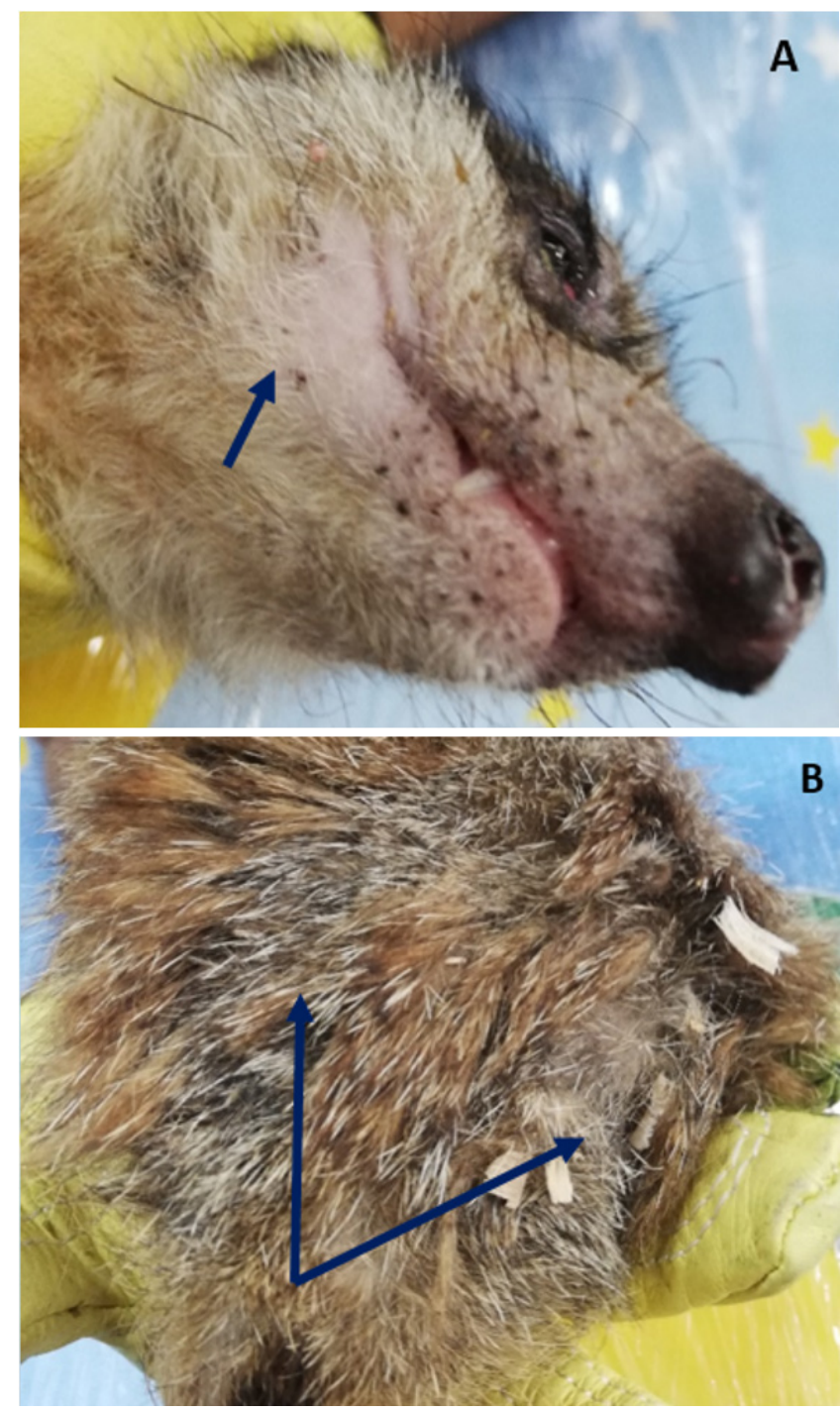

Figura 1. A. La flecha azul indica la alopecia en la comisura labial. B. Las flechas azules indican la alopecia bilateral. 


\section{Ayudas Diagnosticas}

Se realizó un raspado de piel profundo donde se observó ligera afección del ectotrix, presencia de abundantes cilindros foliculares, queratina, levaduras libres y bacterias (cocobacilos).

Hallazgos ultrasonográficos. Utilizando una sonda microconvex multifrecuencia se observó el útero edematoso, con bordes irregulares (Figura 2A). El ovario izquierdo conservaba su tamaño y forma oval, con aspecto alargado, aplanado e hiperecoico, envuelto de una imagen anecoica delimitada por una delgada pared que daba como resultado un refuerzo posterior (Figura 2B), hallazgos que sugirieron ovarios poliquísticos (Figura 2C). El hígado se encontró de tamaño preservado y ecogenicidad aumentada con respecto al órgano de comparación (riñón, Figura 2C) (Figura 2D), hallazgos que sugirieron hígado graso. Los demás órganos evaluados no presentaron cambios ecográficos que indicaran anormalidad.

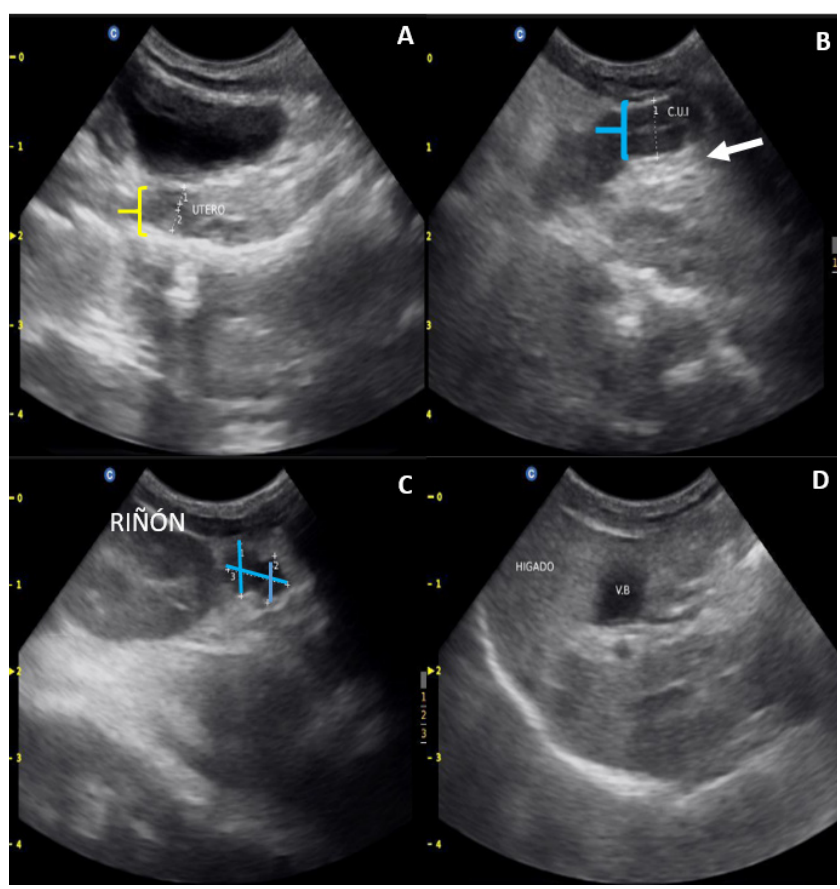

Figura 2. A. El corchete amarillo señala el útero con bordes irregulares. B. El corchete azul señala el ovario izquierdo hiperecoico y la flecha blanca indica el refuerzo posterior. C. Las líneas indican el diámetro de los quistes ováricos. D. Se observa la ecogenicidad aumentada del hígado.

\section{Planes terapéuticos}

Medico. Posterior al diagnóstico se decidió realizar un baño con clorhexidina la cual es usada como bactericida y fungicida, adicional a esto se suministró una dosis única de dipirona (novalgina ${ }^{\circledR}$ ) fármaco analgésico utilizado para el tratamiento de dolor evidenciado en su desplazamiento. El animal quedó en observación hasta el procedimiento quirúrgico.

Quirúrgico. Un mes después del examen clínico y ecográfico se realizó ovariohisterctomía (OVH) utilizando el protocolo anestésico descrito en la tabla 2.

Tabla 2. Protocolo anestésico usado durante el procedimiento y medicamentos usados intra y postoperatorio.

\begin{tabular}{|c|c|c|c|}
\hline Efecto & Vía & Medicamento & $\begin{array}{c}\text { Dosificación } \\
(\mathbf{m g} / \mathbf{k g})\end{array}$ \\
\hline \multirow{3}{*}{ Inducción } & \multirow{3}{*}{ IM } & Ketamina & $5^{*}$ \\
\hline & & Dexmedetomidina & $0.007 *$ \\
\hline & & Midazolam & $0.01 *$ \\
\hline \multicolumn{2}{|c|}{ Mantenimiento Tubo traqueal } & Isoflurano & $4 \% \quad *$ \\
\hline Antibiótico & IV & Cefalotina & 30 \\
\hline AINE & SC & Meloxicam & 0.2 \\
\hline Analgésico & PO & Tramadol & 2 \\
\hline Antibiótico & $\mathrm{PO}$ & $\begin{array}{l}\text { Amoxicilina }+ \\
\text { Ácido Clavulonico }\end{array}$ & 15 \\
\hline AINE & $\mathrm{PO}$ & Meloxicam & 0.1 \\
\hline
\end{tabular}

Se realizó abordaje por la línea media ventral, incidiendo de manera sub-umbilical (Figura 3A) (7), hasta el borde craneal del pubis. El abordaje se realizó de forma más ventral respecto al mismo procedimiento en caninos y felinos, ya que la distribución anatómica de sus órganos reproductivos es más caudal, similar al caso de los mustélidos. Después del abordaje se expuso el útero el cual consiste en un cuerpo corto del que divergen dos cuernos largos y delgados en forma de " $v$ " $(7,8)$.

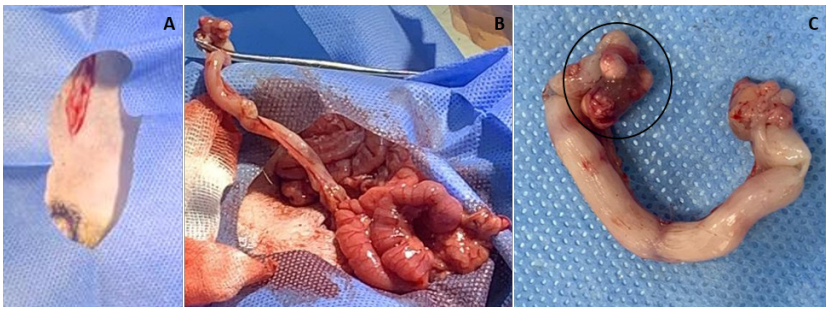

Figura 3. A. Incisión sub-umbilical. B. Exposición de cuernos uterinos. C. El círculo indica el ovario izquierdo con múltiples quistes. 
Al exponer los ovarios, se logró identificar claramente los nódulos, de manera más marcada en el ovario izquierdo (Figura 3BC). Posteriormente se realizó ligadura de los vasos sanguíneos uterinos y transflexión de cuerno uterino para cortar y retirar. Se tomó biopsia escisional de ambos ovarios y parte del cuerno uterino izquierdo, los especímenes fueron remitidos al laboratorio de patología y se diferenciaron con material de sutura. Se administraron líquidos a una tasa de $3 \mathrm{ml} / \mathrm{kg} / \mathrm{h}$, antibiótico de amplio espectro y un AINE intraoperatorio. Se logró la recuperación anestésica satisfactoriamente, sin complicaciones. El animal quedó en tratamiento con antibiótico durante 7 días BID, AINE y analgésico opiode por 3 días SID (Tabla 2).
Hematología. Durante el procedimiento quirúrgico se insertó un catéter intravenoso calibre 24 en la vena cefálica, se tomaron muestras de sangre, en los cuales conforme a la base de datos de la plataforma ZIMS (Zoological Information Management System) en los valores obtenidos se observó disminución del hematocrito, con hemoglobina y glóbulos rojos en rangos normales, anisocitosis, policromasia y recuento de reticulocitos sobre el rango, consistentes con anemia de tipo regenerativo En la química sanguínea se reportó aumento de la relación BUN:Creatinina y proteínas totales, asociado a hiperglobulinemia. Adicionalmente se reportó aumento en la bilirrubina total y en la fosfatasa alcalina (Tabla 1).

Tabla 1. Resultados de hematología y Química sanguínea.

\begin{tabular}{|c|c|c|c|}
\hline Examen & Prueba & Resultados & Valores de referencia \\
\hline \multirow{20}{*}{ Hemograma } & Hto & $25 \%$ & $25.6-54.0$ \\
\hline & $\mathrm{Hb}$ & $8.5 \mathrm{~g} / \mathrm{dl}$ & $7.7-16.6$ \\
\hline & RGR & $5.94 * 10^{\wedge} 6$ & $4.96-12.00$ \\
\hline & VCM & $42 \mathrm{fL}$ & $36.0-52.6$ \\
\hline & $\mathrm{MCHC}$ & $34 \mathrm{~g} / \mathrm{dl}$ & $26.6-36.8$ \\
\hline & Conteo reticulocitos & $11.8 * 10^{\wedge} 3 \mathrm{cells} / \mu \mathrm{L}$ & $1.1-3.8$ \\
\hline & Plaquetas & $779 * 10^{\wedge} 3$ cells $/ \mu \mathrm{L}$ & $39-885$ \\
\hline & RGB & $9.6 * 10^{\wedge} 3$ cells $/ \mu \mathrm{L}$ & $1.6-11.3$ \\
\hline & Neutrófilos \% & $74 \%$ & $26.4-85.0$ \\
\hline & Conteo neutrófilos & $7.1 * 10^{\wedge} 3 \mathrm{cells} / \mu \mathrm{L}$ & $1.35-6.51$ \\
\hline & Bandas \% & $1 \%$ & $0.0-69.9$ \\
\hline & Conteo bandas & $0.1 * 10^{\wedge} 3$ cells $/ \mu \mathrm{L}$ & $0.01-0.21$ \\
\hline & Metamielocitos \% & $0 \%$ & \\
\hline & Conteo metamielocitos & $0 * 10^{\wedge} 3$ cells $/ \mu \mathrm{L}$ & \\
\hline & Linfocitos \% & $25 \%$ & $11.4-72.2$ \\
\hline & Conteo linfocitos & $2.4 * 10^{\wedge} 3$ cells $/ \mu \mathrm{L}$ & $0.6-3.38$ \\
\hline & Monocitos \% & $0 \%$ & $0.0-10.0$ \\
\hline & Conteo monocitos & $0 * 10^{\wedge} 3$ cells $/ \mu \mathrm{L}$ & $0.0-0.5$ \\
\hline & Eosinófilos \% & $0 \%$ & $0.0-5.7$ \\
\hline & Conteo eosinófilos & $0 * 10^{\wedge} 3$ cells $/ \mu \mathrm{L}$ & $0.0-0.3$ \\
\hline \multirow{15}{*}{ Química Sanguínea } & Glucosa & $63 \mathrm{mg} / \mathrm{dl}$ & $46-183$ \\
\hline & BUN & $26 \mathrm{mg} / \mathrm{dl}$ & $14.6-43.6$ \\
\hline & Creatinina & $0,3 \mathrm{mg} / \mathrm{dl}$ & $0.3-1.9$ \\
\hline & BUN: Crea ratio & 102 & $16.0-67.2$ \\
\hline & ALT & $69 \mathrm{U} / \mathrm{L}$ & $35-278$ \\
\hline & AST & $81 \mathrm{U} / \mathrm{L}$ & $37-162$ \\
\hline & Fosfatasa alcalina & $113 \mathrm{U} / \mathrm{L}$ & $4-56$ \\
\hline & Proteína total & $8.2 \mathrm{~g} / \mathrm{dl}$ & $4.8-7.9$ \\
\hline & Albumina & $18 \mathrm{~g} / \mathrm{dl}$ & $20-44$ \\
\hline & $\mathrm{Ck}$ & $121 \mathrm{U} / \mathrm{L}$ & $32-1,101$ \\
\hline & $\mathrm{Ca}$ & $8.4 \mathrm{mg} / \mathrm{dl}$ & $8.0-11.0$ \\
\hline & $P$ & $8.3 \mathrm{mg} / \mathrm{dl}$ & $2.9-8.4$ \\
\hline & Bilirrubina total & $0.7 \mathrm{mg} / \mathrm{dl}$ & $0.0-0.5$ \\
\hline & Colesterol & $378 \mathrm{mg} / \mathrm{dl}$ & $190-717$ \\
\hline & Globulina & $6.4 \mathrm{~g} / \mathrm{dl}$ & $2.1-5.0$ \\
\hline
\end{tabular}


Resultado de la evaluación histopatológica. El ovario izquierdo (con sutura vicryl 2-0) midió $1.3 \mathrm{~cm}$ de longitud mayor y presentaba múltiples nodulaciones de consistencia firme y color amarillento, la de mayor diámetro medía $4 \mathrm{~mm}$. El ovario derecho (sin sutura) midió $1 \mathrm{~cm}$ de diámetro mayor. En todos los cortes del ovario izquierdo, se observó una neoplasia cortical multilobulada, bien delimitada y no encapsulada, que comprimía parcialmente el parénquima y estroma adyacente. Está compuesta por islotes de células poligonales que se distribuyen entre septos finos de colágeno. Las células neoplásicas tienen citoplasmas amplios y finamente vacuolados, con núcleos ovoides, moderadamente cromáticos y homogéneos, que no exhiben atipia ni mitosis. En el ovario derecho se encontró un parénquima ovárico con folículos en diferentes estadíos y un cuerpo lúteo, en los fragmentos de ámpula y cuerno adyacente no se observaron lesiones significativas. (Figura 4).

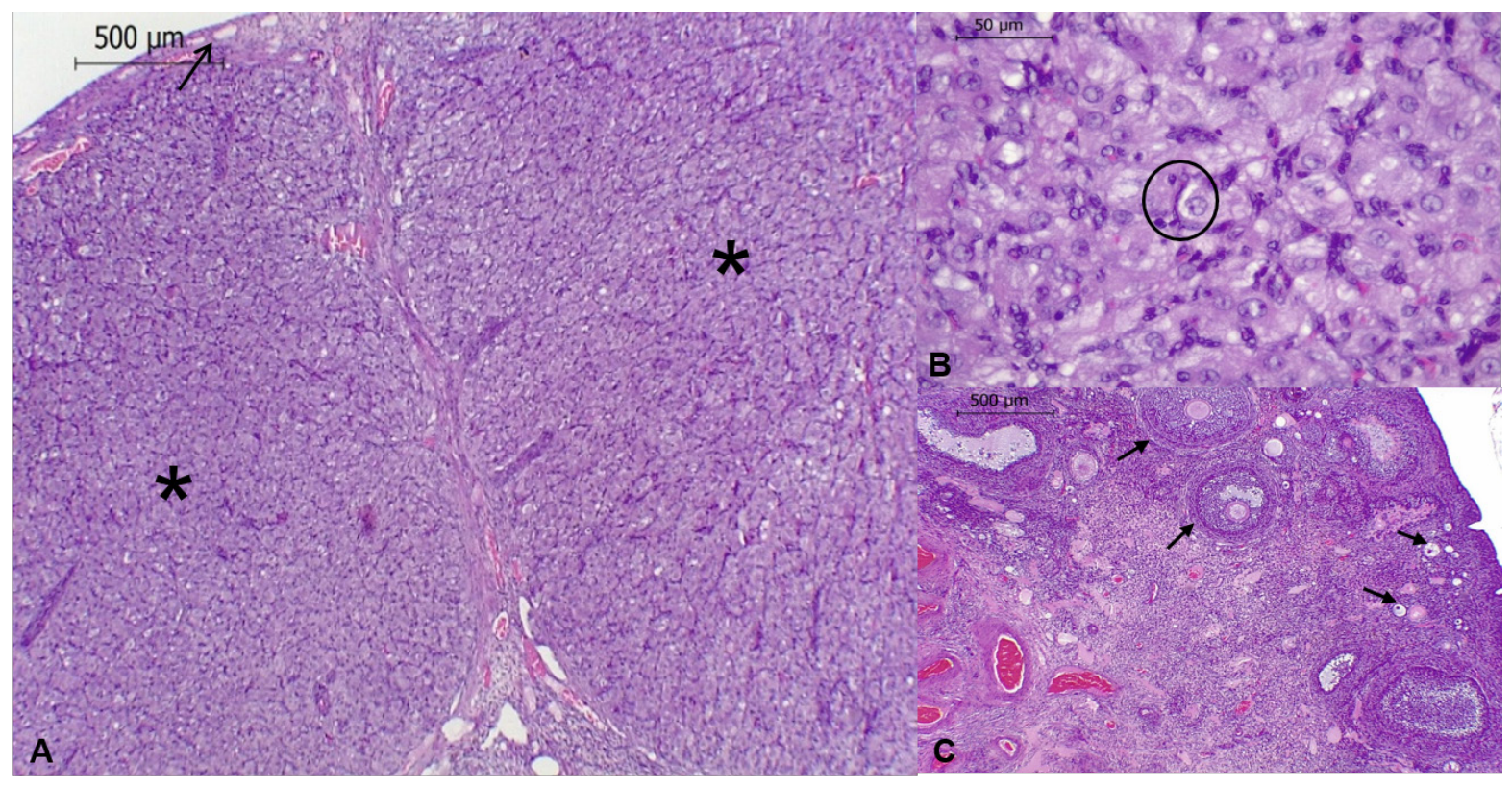

Figura 4. A. La flecha negra indica el desplazamiento del parénquima a causa de la neoplasia (*). Tinción H\&E, 40X B. El circulo muestra las características típicas de las células neoplásicas. Tinción H\&E 400X. C. Las flechas indican los folículos en diferentes estadios. Tinción H\&E 400X.

Evolución. La paciente se logró recuperar satisfactoriamente del procedimiento quirúrgico y los valores de hematología y química sanguínea de seguimiento, realizados un mes y medio posterior a la cirugía se encontraron en rangos normales, además, el animal recuperó su posición normal y la totalidad de su pelaje además de lograr ganancia de peso.

\section{DISCUSIÓN}

La descripción de neoplasias ováricas en pequeños carnívoros silvestres es escasa, usualmente se consideran un hallazgo incidental $y$ por lo general se diagnostican en animales mayores a 5 años de edad (9); sin embargo, en especies afines, como es el caso de los carnívoros domésticos se ha determinado que el grado de incidencia de tumores ováricos no supera el $3.7 \%$ y en el caso específico de los tumores de las células gonadales y del estroma suponen del $34 \%$ de todos los tumores ováricos $(10,11)$. En este caso, se logró determinar la presencia de un Luteoma, el cual es un tumor del estroma de los cordones sexuales, que se origina a partir de células especializadas del ovario, encargadas de la producción de estrógenos y progesterona (12). Los Luteoma son de origen biológico benigno, se presentan generalmente de forma unilateral, hallazgo consistente con lo observado en este caso, usualmente son de aspecto lobulado, de consistencia firme y tienen una coloración blanco-amarillento en la superficie $(10,13)$. Esta neoplasia puede ser hormonalmente activa, cursando con comportamientos reproductivos anormales, tales como anestro persistente, estro intermitente o continuo $\mathrm{y} / \mathrm{o}$ masculinización $(14,15,16)$. En este caso la 
paciente presentó reportes de apareamientos constantes y frecuentes previos al diagnóstico, hallazgos consistentes con lo reportado previamente.

Los tumores estromales del cordón sexual en caninas se asocian con signos de hiperestrogenismo, tales como alopecia de origen endocrino, mielo supresión y anemia no regenerativa, leucopenia, trombocitopenia, diátesis hemorrágicas e hiperplasia endometrial quística $\mathrm{y} / \mathrm{o}$ piómetra $(14,15)$, algunos de estos signos fueron observados en la paciente. Otros diagnósticos diferenciales considerados, además de la alopecia multifocal asociada a hiperestrogenismo y los ovarios poliquísticos, fueron otros tumores ováricos y uterinos como el leiomioma y teratoma, los cuales son frecuentemente reportados en carnívoros domésticos y hurones, a menudo con presentación unilateral $(7,10)$. Previamente en la Fundación Zoológica de Cali, se han diagnosticado leiomiomas uterinos en múltiples Suricatas. Los signos de dolor abdominal descritos en la paciente podrían asociarse con la masa o los cambios uterinos. Finalmente, las alteraciones hematológicas encontradas (aumento del BUN y proteínas totales), fueron relacionadas con una hiperglobulinemia por inflamación crónica.

El incremento de la fosfatasa alcalina y la bilirrubina total observados en la química sanguínea, fue asociado con el aumento de la ecogenicidad del hígado, hallazgo sugestivo de hígado graso (7), el cual es una afección común en suricatas mantenidas bajo cuidado humano (4). La etiología es esta afección se asocia con dietas ricas en grasas y sedentarismo, lo que en el caso de especies afines como mustélidos se relaciona con la alta capacidad metabólica para movilizar rápidamente la grasa visceral y ácidos grasos poliinsaturados, generando así lipidosis hepática (17), y a su vez un aumento de la bilirrubina. Adicionalmente se evidenció una anemia regenerativa, la cual se reafirma con la anisocitosis y la policromasia reportadas en el hemograma; en casos reportados de Luteoma en perras se ha evidenciado en la química sanguínea un aumento en la fosfatasa alcalina e hipercolesterolemia (18).

Una herramienta diagnostica clave en este caso fue la ecografía, a través de la cual se logró determinar la presencia de estructuras compatibles con quistes ováricos. Sin embargo, la histopatología fue concluyente para el diagnóstico definitivo. En conclusión, los reportes de literatura en cuanto a la anatomía y patologías reproductivas presentadas en suricatas son escasos, razón por la cual una comparación de la evolución y los signos clínicos de las afecciones específicas del ovario y sus posibles consecuencias es indeterminada.

Es necesario conocer la anatomía y la fisiología de la reproducción en la especie para así comprender los posibles trastornos del tracto reproductivo y sus órganos asociados. Así mismo, conocer las similitudes con otras especies que permitan extrapolar algunos hallazgos, así como la evolución de sus cuadros de enfermedad. Finalmente, este reporte permite dar a conocer el caso de una neoplasia ovárica en una Suricata que presentaba signos asociados con hiperestrogenismo, lo cual resalta la importancia de la evaluación ecográfica como parte del programa de medicina preventiva en animales en cautiverio, la cual es una técnica diagnóstica accesible y poco invasiva que permite determinar estos problemas reproductivos.

\section{Conflicto de intereses}

Los autores declaran que no existe ningún conflicto de intereses con la publicación de este manuscrito.

\section{Agradecimientos}

Fundación zoológica de Cali, Dr. Luis Alejandro Buitrago, quien realizó la ecografía, Dr. Mauricio Santafe quien realizo la cirugía. 


\section{REFERENCIAS}

1. Jordan N, Do Linh San E. Suricata suricatta. The IUCN Red List of Threatened Species. 2015. https://dx.doi.org/10.2305/IUCN. UK.2015-4.RLTS.T41624A45209377

2. Beke Graw, Marta Manser. Life history patterns and biology of the slender mongoose (Galerella sanguinea) in the Kalahari Desert. J Mammal. 2017; 98(2):332-338. https:// doi.org/10.1093/jmammal/gyw178

3. Maya-Pulgarin D, Gonzalez-Dominguez MS, Aranzazu-Taborda D, Mendoza N, MaldonadoEstrada JG. Histopathologic findings in uteri and ovaries collected from clinically healthy dogs at elective ovariohysterectomy: a cross-sectional study. J Vet Sci. 2017; 18(3):407-414. http://dx.doi.org/10.4142/ jvs.2017.18.3.407

4. Molly E. Church, Karen A. Terio, Keel MK. Procyonidae, Viverridae, Hyenidae, Herpestidae, Eupleridae, and Prionodontidae. Karen A. Terio, Denise Mc Aloose, Judy St. Leger. Pathology of Wildlife and Zoo Animals. Elsevier; 2018. https:// doi.org/10.1016/B978-0-12-805306$\underline{5.00012-2}$

5. Juan-Sallés $C$, Prats $N$, López $S$, Domingo $M$, Marco AJ, Morán JF. Epizootic disseminated toxoplasmosis in captive slender-tailed meerkats (Suricata suricatta). Vet Pathol. 1997; 34(1):1-7. http://dx.doi. org/10.1177/030098589703400101

6. Bongiovann L, Di Girolamo N, Della Salda L, Massimi M, Romanucci M,Selleri P, Sudden death in a captive meerkat (suricata suricatta) with arterial medial and myocardial calcification, Asian Pacific Journal of Tropical Biomedicine. 2016; 6(4):357-359. http:// dx.doi.org/10.1016/j.apjtb.2016.01.009

7. Vladimir Jekl, Karel Hauptman. Reproductive medicine in ferrets. Vet Clin North Am Exot Anim Pract. 2017; 20(2):629-663. https:// doi.org/10.1016/j.cvex.2016.11.016

8. Victoria A.Reproductive system of the dog and cat Part 1 - the female system. Vet Nurs J. 2011; 26(2):43-45. http://dx.doi. org/10.1111/j.2045-0648.2010.00013.x

9. Feldman and Nelson. Canine and feline endocrinology and reproduction. Second edition; Elsevier; 2014.
10. Daniel Salazar C, Rosa Perales C. Diagnóstico Histopatológico de Neoplasias en Tracto Reproductivo de Caninos y Felinos Hembras Realizadas en el Laboratorio de Patología Animal de la Universidad Nacional Mayor de San Marcos (2007-2015). Rev Inv Vet Perú 2017; 28(2):468-475. http://dx.doi. org/10.15381/rivep.v28i2.13068

11. Lourdes Caballero Flores. A propósito de un caso clínico: Tumor ovárico primario. Badajoz Veterinaria. 2017; 7:52-54. https://www.colegioveterinariosbadajoz. com/images/Revistas/2017/Numero7.pdf

12. Corey FS, . Lawrence JA. Tumors of the Female Reproductive System. 5th edition. David Vail Small Animal Clinical Oncology. Elsevier; 2012. https://doi.org/10.1016/ B978-1-4377-2362-5.00026-8

13. Haimerl P, Arlt SP. Cystic ovaries and ovarian neoplasia in the female dog - a systematic review. Reprod Domest Anim. 2016; 51(1):3-11. https://doi.org/10.1111/ $\underline{\text { rda. } 12781}$

14. Darbaz I, Ergene O, Sonmez G, Aslan S. Ovarian Tumour in a Bitch: Diagnosis, Surgery and Recovery. Kafkas Univ Vet Fak Derg. 2017; 23(5):839-842 https://doi. org/10.9775/kvfd.2017.17718

15. Knauf $Y$, Köhler $K$, Knauf $S$, Wehrend $A$. Histological classification of canine ovarian cyst types with reference to medical history. J Vet Sci. 2018; 19(6):725-734. https:// doi.org/10.4142/jvs.2018.19.6.725

16. Dalen WA, MacLachlan J. Tumors of the genital systems. In: Meuten DJ. Tumors in Domestic Animals. fifth edition. John Wiley \& Sons; 2017. https://doi. org/10.1002/9781119181200.ch16

17. Huynh M, Laloi F. Diagnosis of liver disease in domestic ferrets (Mustela putorius). Vet Clin North Am Exot Anim Pract. 2013; 16(1):121-144. http://dx.doi. org/10.1016/j.cvex.2012.10.003

18. Yamini B, VanDenBrink PL, Refsal KR. Ovarian steroid cell tumor resembling luteoma associated with hyperadrenocorticism (Cushing's disease) in a dog. Vet Pathol. 1997; 34(1):57-60. http://dx.doi. org/10.1177/030098589703400112 